\title{
A Case of Advanced Gastroesophageal Junction Cancer with Bulky Lymph Node Metastases Treated with Nivolumab
}

\author{
Naohiko Nakamura Shinichi Kinami Jun Fujita Daisuke Kaida \\ Yasuto Tomita Takashi Miyata Hideto Fujita Nobuhiko Ueda \\ Hiroyuki Takamura Takeo Kosaka
}

Department of Surgical Oncology, Kanazawa Medical University, Kahoku, Japan

Keywords

Gastroesophageal junction cancer · Nivolumab · Adenocarcinoma

\begin{abstract}
A 71-year-old woman was diagnosed with advanced gastroesophageal junction cancer with bulky lymph nodes along the cardiac region and the lower mediastinum (GE-Circ type 3 T3 $\mathrm{N} 3 \mathrm{MO} \mathrm{HO}$ stage III) and received treatment with S-1 and oxaliplatin (SOX) as first-line chemotherapy. After 3 cycles of SOX, severe anorexia and diarrhea were observed. We converted from this regimen of systemic chemotherapy to ramucirumab (RAM) monotherapy as secondline chemotherapy. This treatment resulted in a reduction in size of the metastatic lymph nodes along the cardiac region and the lower mediastinum. However, progression of lymph node metastasis and the primary tumor was observed following 7 months of RAM monotherapy. Therefore, nivolumab was initiated as third-line chemotherapy 14 months after the initial treatment. After 3 months of nivolumab administration, a 47\% reduction in metastatic lymph nodes was achieved and a regression of the primary gastric tumor as seen on an enhanced computed tomography scan. After 7 months of nivolumab monotherapy, the diameter of the target lymph nodes had reduced by $81 \%$ from baseline, and there was no evidence of malignancy upon pathological assessment of the primary tumor site biopsy. The patient survived with nivolumab monotherapy for approximately 2 years after her first visit, without any adverse reaction to nivolumab.




\section{Introduction}

Gastric cancer (GC) is the second leading cause of cancer-related mortality and the fourth most commonly diagnosed malignant disease [1]. Systemic chemotherapy is the standard treatment for recurrent or metastatic GC [2]. Although some chemotherapy regimens have been shown to improve the survival outcomes of advanced GC patients, the prognosis remains poor and further therapeutic development is needed. Under these conditions, nivolumab, a fully human IgG4 monoclonal antibody against programmed death-1 (PD-1), has been approved for use in advanced GC, including gastroesophageal junction cancer (GEJC) as a third-line chemotherapeutic agent, in Japan, and is expected to improve the prognosis of advanced GC patients. On the other hand, adenocarcinoma of the gastroesophageal junction, which is a representative malignancy located between the esophagus and stomach, is well known to have unique clinicopathological features and biological behaviors. It has been reported that the incidence of GEJC has gradually increased worldwide in recent years $[3,4]$, and the prognosis of GEJC is worse than that of distal GC [5].

Here, we present a case of advanced GEJC with bulky lymph node metastases that was successfully treated with nivolumab and resulted in an improved prognosis.

\section{Case Presentation}

A 71-year-old woman (height, $142 \mathrm{~cm}$; body weight, $49.0 \mathrm{~kg}$; Eastern Cooperative Oncology Group performance status, 1) who presented with vomiting was diagnosed with type III advanced GC of the gastroesophageal junction (Siewert type II [6]) using an upper gastrointestinal endoscopy (Fig. 1). Concurrently, an enhanced computed tomography (eCT) scan revealed thickening of the upper gastric wall and enlarged lymph nodes along the cardiac region and lower mediastinum (Fig. 2). Lung and liver metastases were not observed on eCT.

Biopsy results from the gastric tumor indicated a diagnosis of poorly differentiated adenocarcinoma, and the HER2 status was negative, as assessed by immunohistochemistry. The clinical diagnosis was GE-Circ type $3 \mathrm{~T} 3 \mathrm{~N} 3 \mathrm{M} 0 \mathrm{H} 0$ stage III (according to the 15th edition of the Japanese Classification of Gastric Cancer [7]). For this patient, we chose S-1 (100 mg/ day) and oxaliplatin $\left(100 \mathrm{mg} / \mathrm{m}^{2}\right)$ as chemotherapeutics (SOX) because curative resection was considered to be an excessively invasive procedure with thoracotomy. After 3 cycles of SOX, severe anorexia and diarrhea (grade 3; National Cancer Institute Common Terminology Criteria for Adverse Events version 3.0) were observed despite a dose reduction in S-1 and oxaliplatin. Therefore, we converted the regimen from systemic chemotherapy to ramucirumab (RAM) monotherapy.

Fig. 1. Primary tumor as seen on upper gastrointestinal endoscopy before treatment. Type III advanced adenocarcinoma was observed in the gastroesophageal junction.

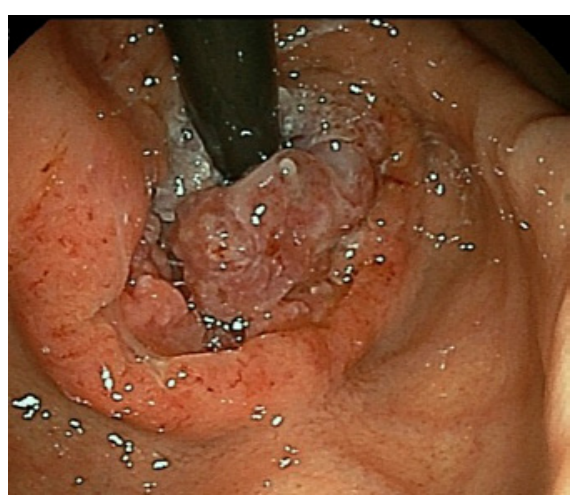



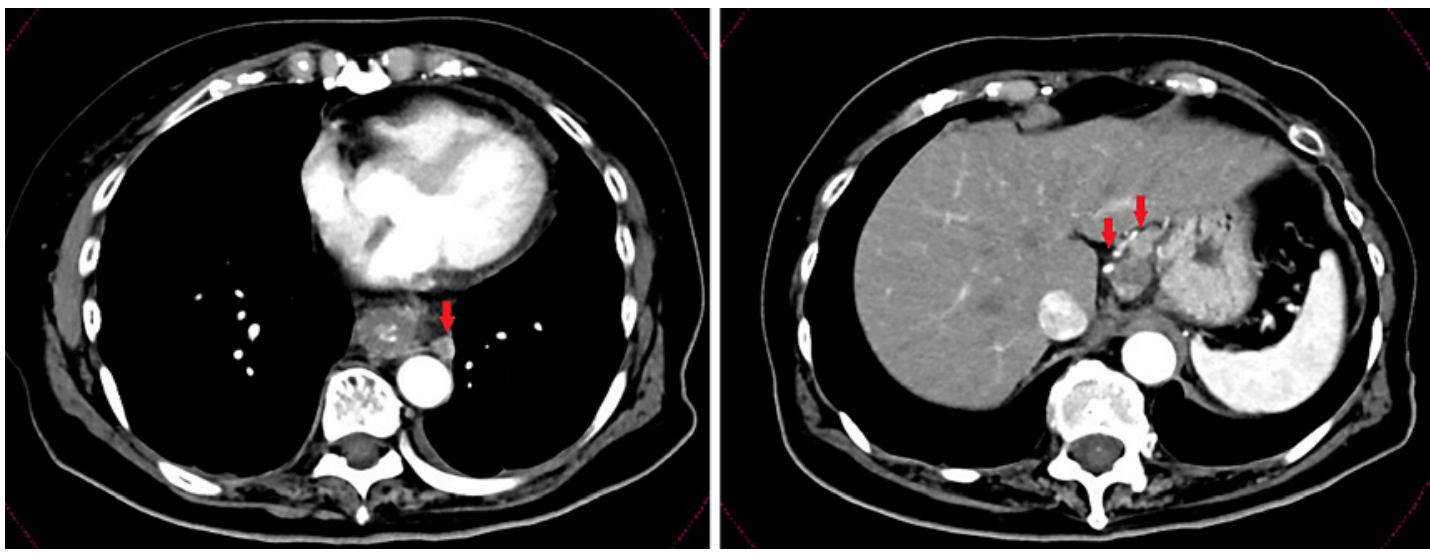

Fig. 2. Enlarged lymph nodes along the cardiac region and lower mediastinum on enhanced computed tomography before treatment (arrows).

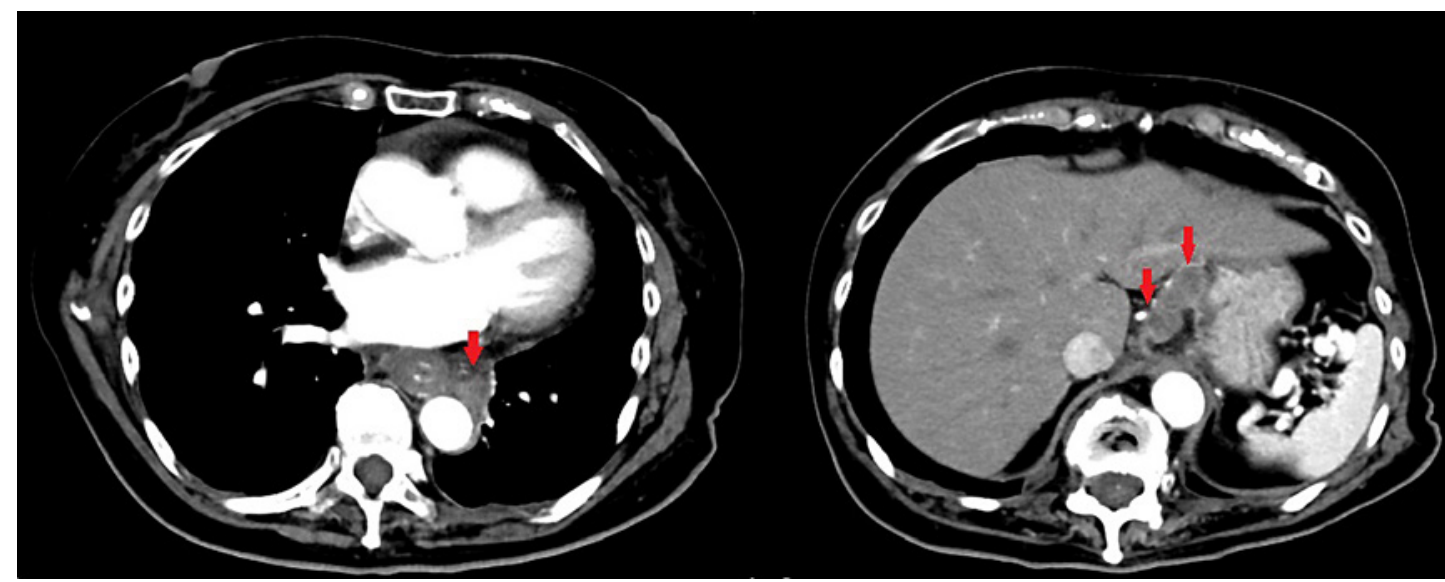

Fig. 3. Progression of the metastatic lymph nodes along the cardiac region and lower mediastinum as seen on an enhanced computed tomography scan after ramucirumab monotherapy (arrows).

Four months after the start of RAM monotherapy, the diameter of the metastatic lymph nodes was slightly decreased, but the tumor was still stable according to RECIST version 1.1 [8]. An eCT following 7 months of RAM monotherapy revealed progression of both metastasis in the lymph nodes and the primary tumor in the stomach (Fig. 3), and we initiated nivolumab monotherapy as third-line chemotherapy. Three months of nivolumab administration ( 7 cycles) led to a $47 \%$ reduction in the metastatic lymph nodes, and no new metastatic lesions were seen on eCT. After 7 months of nivolumab monotherapy (a total of 14 cycles of nivolumab were administered), the diameter of the target lymph nodes showed an $81 \%$ reduction from baseline and each had reduced to less than $10 \mathrm{~mm}$ in size (Fig. 4). Although the primary lesion of the gastroesophageal junction was still present as seen with an upper gastrointestinal endoscopy (Fig. 5), there was no evidence of malignancy from pathological assessment with a biopsy of the primary tumor. The patient did not exhibit any adverse events and has survived with nivolumab monotherapy for approximately 2 years from her first visit. 


\section{Case Reports in \\ Oncology}

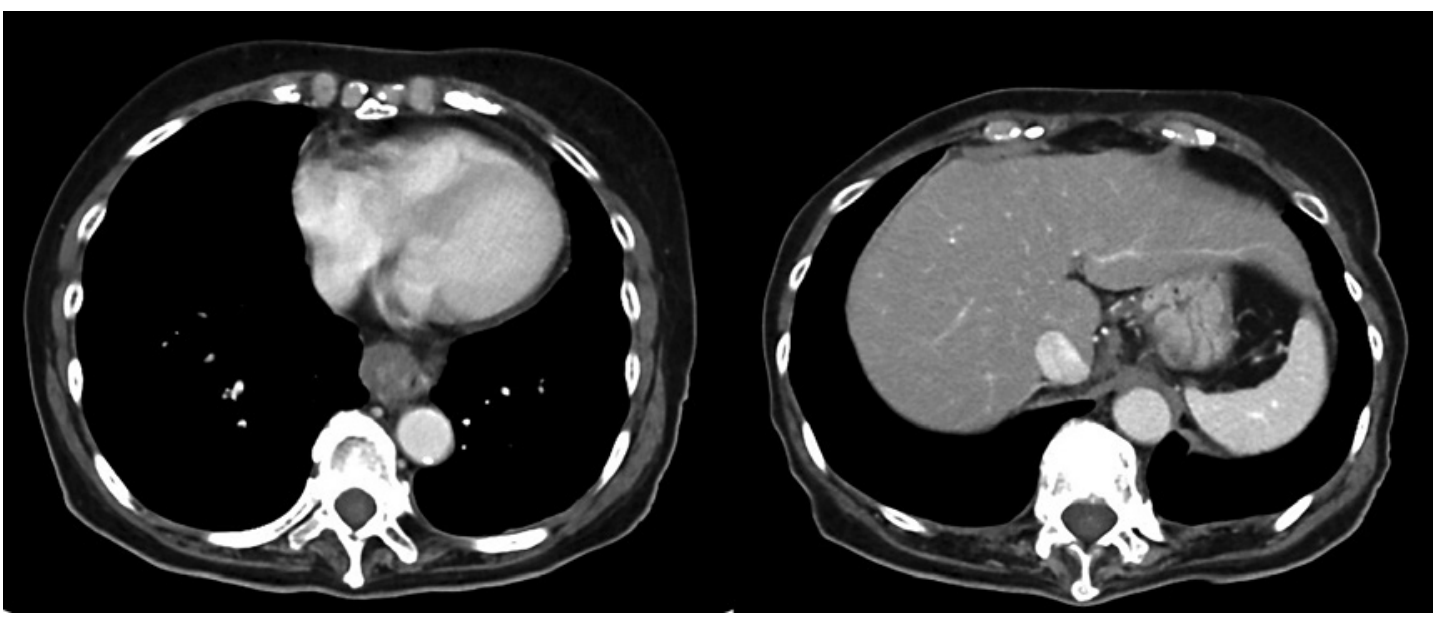

Fig. 4. The diameters of the metastatic lymph nodes were reduced on enhanced computed tomography after 7 months of nivolumab administration.

Fig. 5. The primary lesion of the gastroesophageal junction was still present as seen with an upper gastrointestinal endoscopy. There was no evidence of malignancy from pathological assessment with a biopsy of the primary tumor.

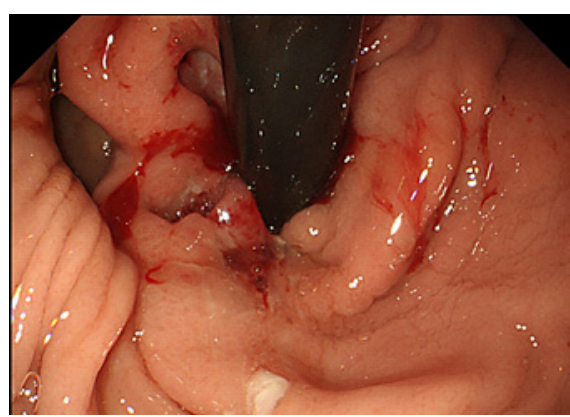

\section{Discussion}

Immune checkpoint inhibitors are now widely used in many types of cancer. For advanced GC, nivolumab monotherapy has been shown to be more effective for pretreatment of GC patients in the ATTRACTION-2 study in 2017 [9]. The ATTRACTION-2 study indicated a median progression-free survival of 1.16 months and a median overall survival of 5.26 months with nivolumab monotherapy. The objective response rate was $11.2 \%$, and the proportion of patients who showed a complete response was $0 \%$. Our case showed a significant reduction in the diameter of the metastatic lymph nodes, but the primary lesion persisted, albeit at a reduced size. Although a biopsy from the primary tumor lesion did not reveal evidence of malignancy, the response to nivolumab monotherapy was considered only a partial one according to RECIST. It is difficult to predict a tumor response to nivolumab administration. A recent report has indicated a better prognosis in advanced GC patients with a low neutrophilto-lymphocyte ratio (NLR) before the first cycle of nivolumab monotherapy [10]. This case also had a low NLR before the initiation of nivolumab and had a better resulting prognosis due to tumor regression after nivolumab monotherapy. Therefore, the NLR might be useful for predicting tumor response to nivolumab monotherapy.

In addition to advanced GC, surgery is the primary curative modality for esophagogastric junction cancer (EGJC). Even if a curative resection is achieved, the overall prognosis of EGJC 
remains poor because of distant and local regional recurrence, with 5-year survival rates averaging approximately 30\% [11]. Thus, perioperative chemotherapy for advanced EGJC patients may be important for improving survival. In this case, Siewert type II adenocarcinoma with bulky lymph node metastases was considered a borderline candidate for resection using surgery alone, and we administered systemic chemotherapy aiming for a curative resection by gastrectomy following chemotherapy. However, SOX was the first-line chemotherapy and it cannot be continuously administered due to its severe adverse effects. RAM monotherapy, used as second-line treatment, resulted in stable disease as the tumor response. In addition, this case exhibited bulky lymph nodes along the lower mediastinum, and thoracotomy was needed for a curative resection. We initiated nivolumab monotherapy as third-line therapy instead of using a curative resection. As a result of nivolumab monotherapy, the patient maintained a good nutritional condition and daily living standards and survived for 8 months with nivolumab monotherapy.

In conclusion, we experienced a case of advanced EGJC that showed a remarkable clinical response and obtained a better prognosis with nivolumab monotherapy. It is essential to accumulate clinical outcomes of nivolumab monotherapy for advanced EGJC.

\section{Statement of Ethics}

We obtained written informed consent from the patient. The research reported in this paper was in compliance with the Helsinki Declaration.

\section{Disclosure Statement}

The authors declare that they have no competing interests.

\section{Funding Sources}

All authors did not receive any funding for this paper.

\section{Author Contributions}

N.N. reported the case and wrote the manuscript. S.K., J.F., D.K., Y.T., T.M., H.F., N.U., and T.K. were involved in treating the patient. S.K. and T.K. participated in critically revising the manuscript. All authors have read and approved the final manuscript.

\section{References}

1 Jemal A, Center MM, DeSantis C, Ward EM. Global patterns of cancer incidence and mortality rates and trends. Cancer Epidemiol Biomarkers Prev. 2010;19(8):1893-907.

2 Japanese Gastric Cancer Association. Japanese Gastric cancer treatment guidelines 2014 (ver. 4). Gastric Cancer. 2017;20(1):1-19.

3 Steevens J, Botterweck AA, Dirx MJ, van den Brandt PA, Schouten LJ. Trends in incidence of oesophageal and stomach cancer subtypes in Europe. Eur J Gastroenterol Hepatol. 2010;22(6):669-78.

4 Kusano C, Gotoda T, Khor CJ, Katai H, Kato H, Taniguchi H, et al. Changing trends in the proportion of adenocarcinoma of the esophagogastric junction in a large tertiary referral center in Japan. J Gastroenterol Hepatol. 2008;23(11):1662-5. 
5 Liu K, Zhang W, Chen X, Chen X, Yang K, Zhang B, et al. Comparison on clinicopathological features and prognosis between esophagogastric junctional adenocarcinoma (Siewert II/III types) and distal gastric adenocarcinoma: retrospective cohortstudy, a single institution, high volume experience in China. Medicine (Baltimore). 2015;94(34):e1386.

6 Siewert JR, Stein HJ, Feith M. Adenocarcinoma of the esophago-gastric junction. Scand J Surg. 1999;95(4): 260-9.

7 Japanese Gastric Cancer Association. Japanese classification of gastric carcinoma. 15th ed. Tokyo: Kanehara; 2017.

8 Therasse P, Arbuck SG, Eisenhauer EA, Wanders J, Kaplan RS, Rubinstein L, et al. New guidelines to evaluate the response to treatment in solid tumors. European Organization for Research and Treatment of Cancer, National Cancer Institute of the United States, National Cancer Institute of Canada. J Natl Cancer Inst. 2000; 92(3):205-16.

9 Kang YK, Boku N, Satoh T, Ryu MH, Chao Y, Kato K, et al. Nivolumab in patients with advanced gastric or gastrooesophageal junction cancer refractory to, or intolerant of, at least two previous chemotherapy regimens (ONO-4538-12, ATTRACTION-2): a randomised, double-blind, placebo-controlled, phase 3 trial. Lancet. 2017; 390(10111):2461-71.

10 Ogata T, Satake H, Ogata M, Hatachi Y, Inoue K, Hamada M, et al. Neutrophil-to-lymphocyte ratio as a predictive or prognostic factor for gastric cancer treated with nivolumab: a multicenter retrospective study. Oncotarget. 2018;9(77):34520-7.

11 Njei B, McCarty TR, Birk JW. Trends in esophageal cancer survival in United States adults from 1973 to 2009: a SEER database analysis. J Gastroenterol Hepatol. 2016;31(6):1141-6. 